614. L. Claisen und E. Haase: Umlagerung des Acetophenon$O$-Benzoats in Dibenzoylmethan.

[Mittheilung aus dem chem. Institut der Universität Kiel.]

(_ingegangen am 13. October; mitgetheilt in der Sitzung von Hrn. O. Diel s.)

In früheren Arbeiten ${ }^{1}$ ) baben wir uns mit den $O$-Acylaten der 1.3-Ketonsäureester und der 1.3-Diketone beschäftigt. Zunächst fanden wir, dass diese Körper, als deren einfachsten Vertreter man den $O$. acetylirten Acetessigester,

$\mathrm{CH}_{3} . \mathrm{C}\left(\mathrm{O} . \mathrm{CO} . \mathrm{CH}_{3}\right): \mathrm{CH} . \mathrm{COOC}_{2} \mathrm{H}_{5}$,

betrachten kann, sehr leicht und glatt durch Behandlung des betreffenden Ketonsänreesters oder Diketons mit Säurechloriden bei Gegenwart von tertiären Basen (Pyridin, Dimethylanilin, Antipyrin u. s. w.) zu erbalten sind. Sodann gelang es uns, diese $O$.Acylderivate durch gelindes Erwärmen mit alkalischen Agentien in die Alkalisalze der isomeren C-Acylderivate umzulagern, z. B.:

$$
\begin{aligned}
\mathrm{CH}_{3} \cdot \mathrm{C}\left(\mathrm{O} \cdot \mathrm{CO} \cdot \mathrm{CH}_{3}\right) & : \mathrm{CH} . \mathrm{COOC}_{2} \mathrm{H}_{3} \\
& =\mathrm{CH}_{3} \cdot \mathrm{C}(\mathrm{OH}): \mathrm{C}\left(\mathrm{CO} \cdot \mathrm{CH}_{3}\right) \cdot \mathrm{COOC}_{2} \mathrm{H}_{3} .
\end{aligned}
$$

Von vornberein war es wahrscheinlich, dass die entsprechende Umlagerung sich auch bei den $O$-acylirten Ketonen wärde ansführen lassen. Hatte der Eine von nns ${ }^{2}$ ) doch für die $O$-alkylirten Ketone schon vor längerer Zeit festgestellt, dass sie beim Ueberhitzen in die $C$-alkylirten Ketone äbergehen; $O$-Aethyl-Acetophenon z. B. lagerte sich beim Kochen unter Druck ziemlich vollständig in $C$-Aethyl-Acetophenon, also in Butyrophenon, um:

$$
\mathrm{C}_{6} \mathrm{H}_{5}, \mathrm{C}\left(\mathrm{OC}_{2} \mathrm{H}_{3}\right): \mathrm{CH}_{2}=\mathrm{C}_{4} \mathrm{H}_{5} \cdot \mathrm{CO} \cdot \mathrm{CH}_{2} \cdot \mathrm{C}_{2} \mathrm{H}_{5} \text {. }
$$

Dem entsprechend durfte auch bei den $O$-acylirten Ketonen eine leichte Umwandelung in die $C$-acylirten erwartet werden; $O$ Benzoyl-Acetophenon z. B. musste sich leicht in C-Benzoyl-Acetophenon, d. h. in Dibenzoylmethan, umlagern lassen:

$$
\mathrm{C}_{6} \mathrm{H}_{5} . \mathrm{C}\left(\mathrm{O} . \mathrm{CO} . \mathrm{C}_{6} \mathrm{H}_{5}\right): \mathrm{CH}_{2}=\mathrm{C}_{6} \mathrm{H}_{6} \cdot \mathrm{CO} . \mathrm{CH}_{2} \cdot \mathrm{CO} . \mathrm{C}_{6} \mathrm{H}_{5} \text {. }
$$

Indessen waren solche Keton. O-Acylate damals noch nicht bekannt. Erst neuerding ${ }^{3}$ hat $\mathrm{Lees}^{3}$ ) die interessante Beobachtung ge-

1) Claisen, Ann. d. Chem. 291, 106 und 110; 297, 2 (Anmerk.); Claisen und Haase, diese Berichte 33, 1242 und 3778 [1900]. Jeber Anwendung unseres Verfahrens der $O$-Acylirung der Ketonsăureester nach der Pyridinmethode vergl. auch Bouveault, Bull. Soc. chim. 27, 1160.

2) Diese Berichte 29, 2931 [1896].

3) Lees, Journ. Chem. Soc. 1903, 145; von einigen Aldehyden hat Lees ein gleiches Verhalten gegen Benzoylchlorid constatirt. 
macht, dass einzelne hochsiedende Ketone (Methylnonylketon, Acetophenon a. s. w.) durch fortgesetztes Kochen mit Benzoylchlorid in derartige $O$-Benzoate, R.C $\left(\mathrm{O} . \mathrm{CO} . \mathrm{C}_{6} \mathrm{H}_{5}\right): \mathrm{CH}_{2}$, verwandelt werden. Einen von diesen Körpern, das A cetophenon-O-Benzoat, baben wir nun zu einem diesbezüglichen Versuch benutzt und festgestellt, dass die erwartete Umlagerung bei ibm in der That ziemlich leicht eintritt.

Acetophenon-O-Benzoat, $\mathrm{C}_{6} \mathrm{H}_{3} \cdot \mathrm{C}\left(\mathrm{O} \cdot \mathrm{CO} \cdot \mathrm{C}_{6} \mathrm{H}_{5}\right): \mathrm{CH}_{2}$.

Diese Verbindung, welche Lees als ein Oel beschreibt, warde von uns in krystallisirtem Zustand erhalten. Den Angaben von Lees. folgend, liessen wir $200 \mathrm{~g}$ Acetophenon mit der berechneten Menge Benzoylchlorid 10 Stunden lang unter Rückfuss lebbaft sieden und destillirten dann das unverändert gebliebene Keton und Säurecblorid unter vermindertem Drucke ab. Bei erneutem Kochen dieses Vorlaufs für sich trat wieder ziemlich viel Salzsäure auf, und ein weiteres. Quantum von dem Benzoat wurde gebildet. Nachdem dies einige Male wiederholt worden war, betrug der gesammte höher siedende Rückstand $245 \mathrm{~g}$. Zur Entfernung von möglicher Weise mitentstandener Benzoësäure lösten wir das Oel in Aether und schüttelten es uit wässrigem Natriumcarbonat unter Eiskühlung gat durch. Dann. wurde der Aether entfernt und das Oel bei $11 \mathrm{~mm}$ Druck rectificirt. Ein beträchtlicher Theil ging bei $190-200^{\circ}$ über, das Meiste davon bei $192-194^{\circ}$. Aus dieser letzteren Fraction, einem hellgelben Oel, schieden sich bei längerem Stehen in der Winterkälte prächtige, farblose, fächenreiche Krystalle ron dicktafeligem Habitus ab. Sie wurden abgesaugt, aus niedrig siedendem Ligroön amkrystallisirt und schmolzen dann bei $41^{\circ}$. Die Analyse ergab genaue Uebereinstimmung mit der von Lees für das Oel ermittelten Formel $\mathrm{C}_{15} \mathrm{H}_{12} \mathrm{O}_{2}$ :

$0.2472 \mathrm{~g}$ Sust: $0.7278 \mathrm{~g} \mathrm{CO}_{2}, 0.1208 \mathrm{~g} \mathrm{H}_{2} \mathrm{O}$.

$$
\begin{aligned}
& \mathrm{C}_{15} \mathrm{H}_{19} \mathrm{O}_{2} \text {. Ber C 80.36, H } 5.36 . \\
& \text { Gef. " 80.29, } 5.43 .
\end{aligned}
$$

Die Verbindung kann leicht wieder in ihre Componenten gespalten werden: in benzoësaures Kalium und Acetophenon durch Erwärmen nnit wässrig-alkoholischem Kali; in Benzoëester und Acetophenon durch Erhitzen im Rohr mit Alkohol, dem etwas Natriumäthylat zugesetzt ist.

Weniger bewährte sich für die Darstellung des Acetophenon- $O$ Benzoats die Behandlung mit Benzoylchlorid und Pyridin, die uns bei der $O$ Benzoylirung der Ketonsäureester so gute Resultate ergeben hatte. Die Mischung von Keton, Säurechlorid und Pyridin (von Letzterem mehr als die berechnete Menge) wurde längere Zeit auf dem Wasserbade erwärmt und dann, zur Beseitigung des entstondenen salzsauren Pyridins, mit Aether extrabirt. Den ätberischen Auszug 
wuschen wir successive mit verdünnter Salzsäure und mit Sodalösung, trockneten ihn, dunsteten den Aether ab und unterwarfen das rückständige Oel der Rectification. Ein nicht unbeträchtlicher Theil, etwa tie Hälfte rom Gewicht des angewandten Acetophenons, ging unter $10 \mathrm{~mm}$ Druck bei $190-203^{\circ}$ über; ein schärferer Siedepunkt wollte sich aber nicht einstellen, und ebenso wenig gelang es, das Oel zum Erstarren zu bringen. Augenscheinlich waren andere Körper (vielleicht Condensationsproducte des Acetophenons für sich) mit entstanden, welche die Reinabscheidung des $O$-Benzoats erschwerten.

Eine eigenthümliche Substanz ergab sich, als wir die Mischung von Acetophenon, Benzoylchlorid und Pyridin bei gewöhnlicher Temperatur 6-7 Wochen lang stehen liessen. Auch hier wurde die dunkelbräunliche, durch ausgeschiedenes Pyridinchlorhydrat halbfeste Reactionsmasse so lange mit Aether digerirt, bis nichts mehr in denselben einging; darauf wurde der im Sonstigen wie oben behandelte ätherische Auszug freiwillig verdunsten gelassen. Aus dem verbleibenden Oel schieden sich gelbe Krystalle ab, welche durch Waschen mit etwas kaltem Methylalkohol und Umkrystallisiren aus heissem Methylalkohol leicht za reinigen waren. Aus $100 \mathrm{~g}$ Acetophenon wurden $15 \mathrm{~g}$ von diesem Product gewonnen. Die Analyse ergab die Formel $\mathrm{C}_{20} \mathrm{H}_{17} \mathrm{NO}_{2}$, der Körper war also durch Zusammentritt von je einem Molekül Acetophenon, Benzoylehlorid und Pyridin entstanden:

$$
\begin{aligned}
& \mathrm{C}_{6} \mathrm{H}_{3} \cdot \mathrm{CO} \cdot \mathrm{CH}_{3}+\mathrm{Cl} . \mathrm{CO} \cdot \mathrm{C}_{6} \mathrm{H}_{5}+2 \mathrm{C}_{5} \mathrm{H}_{5} \mathrm{~N} \\
& =\mathrm{C}_{20} \mathrm{H}_{17} \mathrm{NO}_{2}+\mathrm{C}_{5} \mathrm{H}_{5} \mathrm{~N} \cdot \mathrm{HCl} \text {. } \\
& \mathrm{C}_{20} \mathrm{H}_{47} \mathrm{NO}_{2} \text {. Ber. } \mathrm{C} 79.20, \mathrm{H} 5.61, \mathrm{~N} 4.62 \text {. } \\
& \text { Gef. " 79.19, } \$ 5.81, \text { \$ } 5.05 \text {. }
\end{aligned}
$$

Die Substanz bildet gelbe, kurze Prismen, schmilat bei $110^{\circ}$ und zersetzt sich oberhalb $230^{\circ}$ unter Aufsieden und Verbreitung intensiven Pyridingeruchs. Sie ist unlöslich in Wasser, leicht lốslich in Chloroform, schwer löslich in Aether, gut umkrystallisirbar aus hoissem Methyl- und Aethyl-Alkohol. Auffallender Weise wird die gelbe, alkoholische Lösung beim Kocben, namentlich auf Zugabe vou etwas Wasser, nabezu farblos, wie auch die daraus sich abscheidende Krystallisation fast rein weiss ist; nach dem Abfiltriren und Trocknen gewinnen die Kryställchen aber bald die gelbe Farbe zurück. Eine Aenderung des Schmelzpunktea ist mit diesem rorübergehenden Farbwechsel nicht rerbunden.

Durch Kochen mit verdünuter Schwefelsäure wird die Substanz sehr rasch in Acetophenon, Benzoësüure und schwefelsaures Pyrilin gespalten. Beim Erwärmen mit concentrirter Salcsäure tritt deutlich der Geruch nach Benzoylchlorid auf Der Körper zerfällt also leicht 
in die Componenten, aus denen er sich gebildet bat, zurück. Seiner Constitution nach könnte er eine Verbindung des Acetophenon-O-Benzoats mit einem Molekül Pyridin sein; indessen haben Versuche, ihn aus diesen beiden Bestandtheilen zusammenzusetzen, bis jetzt noch keinen Erfolg gehabt.

Das Verhalten dieses eigenthümlicben Additionsproductes soll noch eingehender untersucht werden; wir boffen, dadurch einige Aufklärung za gewinnen über die Rolle, welche das Pyridin bei der $O$ dcylirung der Ketonsäureester nach dem früher von uns mitgetheilten Verfahren spielt.

Umlagerung des Acetophenon-O-Benzoats in Dibenzoylmethan. $10 \mathrm{~g}$ von dem $O$-Benzoat worden in dem 7 -fachen Gewicht Benzol gelöst und nach Zugabe der berechneten Menge drahtförmigen Natriums auf dem Wasserbade erwärmt. Um die Reaction einzuleiten, wurde etwas Acetophenon zugesetzt. Nach einigen Stunden war das Natrium verschwunden und hatte sich Dibenzoylmethannatrium in reichlicher Menge als gelblich-weisser Brei abgeschieden. Das Salz wurde dorch Zufügen ron Wasser gelöst und die Benzolschicht noch einige Male erst mit Wasser und dann mit sehr verdünnter Natronlauge ausgeschüttelt $\left.{ }^{1}\right)$. Aus den vereinigten wässrigalkalischen Lösungen wurde das Dibenzoylmethan mittels Kohlensäure gefällt. Nach Umkrystallisiren aus heissem Methylalkohol schmolz es bei $78^{\circ}$ und zeigte alle ihm zakommenden Reactionen (Rothfärbung mit Eisenchlorid, Fällbarkeit durch Kupferacetat, Umwandelung durch Hydroxylamin in Diphenylisoxazol rom Schmp. 140-141 ${ }^{\circ}$ ) in so charakteristischer Weise, dass von der Analyse Abstand genommen werden konnte. Die Ausbeute betrug circa $50 \mathrm{pCt}$. der Theorie.

Von dem Vorgang bei dieser Umlageruug und von der Rolle, welche der Zusatz der kleinen Menge Acetophenon dabei spielt, kann man sich unter Anlehnung an die Ansicht Claisen's über die Bildung des Natracetessigesters ${ }^{2}$ ) folgende Vorstellung machen. Das zugefügte kleine Quantum Acetophenon wird zunächst in Acetophenonnatrium, $\mathrm{C}_{6} \mathrm{H}_{5} . \mathrm{C}(\mathrm{ONa}): \mathrm{CH}_{8}$, verwandelt, und Letzteres lagert sich an das Carbonyl des Benzoyls des Acetophenon-O-Benzoats an:

$$
\begin{aligned}
& \mathrm{C}_{6} \mathrm{H}_{3} . \mathrm{C} \leqslant \mathrm{O} . \mathrm{C}\left(\mathrm{C}_{6} \mathrm{H}_{5}\right): \mathrm{CH}_{2}+\mathrm{NaO} \cdot \mathrm{C}\left(\mathrm{C}_{6} \mathrm{H}_{5}\right): \mathrm{CH}_{2}
\end{aligned}
$$

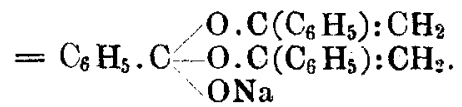

1) Vergl. hierzu Claisen, Ann. d. Chem. 291, 52.

3) Diese Berichte 20,651 [1857]; 21, 1132, 1151 [1888]. - Ann. d. Chem. 281,312 und $329 ; 297,92$. 
Alsdann klappt sich nach einem ähnlichen Mecbanismus wie bei dem Uebergang des phenolkohlensauren Natriums in salicylsaures Natrium:

$$
\mathrm{CO}<{ }_{\mathrm{ONa}}^{\mathrm{OC}_{6} \mathrm{H}_{3}}=\mathrm{CO}<\underset{\mathrm{ONa}}{\mathrm{C}_{6} \mathrm{H}_{4} . \mathrm{OH}}
$$

der eine der beiden sauerstoffgebundenen Acetophenonreste um unter Kohlenstoff bindung und Bildung von:

$$
\mathrm{C}_{6} \mathrm{H}_{3} . \mathrm{C} \underset{\mathrm{ONa}}{\stackrel{\mathrm{ON}}{\mathrm{O}}\left(\mathrm{C}_{6} \mathrm{H}_{6}\right): \mathrm{CH}_{2} .}
$$

Aus diesem letzteren Complex wird durch Abspaltung von Acetophenon (das nun auf's neue durch das vorhandene Natrium in Acetophenonnatrinm verwandelt wird und so wieder in die Reaction eintritt), schliesslich Natrium-Dibenzoylmethan erzeugt ${ }^{1}$ ):

$$
\begin{aligned}
& \mathrm{C}_{6} \mathrm{H}_{3} . \mathrm{C} \underset{\mathrm{ONa}}{\mathrm{O} . \mathrm{C}\left(\mathrm{C}_{6} \mathrm{H}_{3}\right): \mathrm{CH}_{2}=\mathrm{C}_{6} \mathrm{H}_{5} . \mathrm{C}_{\mathrm{ONa}}^{\mathrm{CH}} \cdot \mathrm{CO} \cdot \mathrm{C}_{6} \mathrm{H}_{5}} \\
& +\mathrm{C}_{6} \mathrm{H}_{3} \cdot \mathrm{CO} . \mathrm{CH}_{\mathrm{y}} \text {. }
\end{aligned}
$$

Im Anschlusse an din vorbeschriebenen Versuche hat Hr. Dr. A. Behre ${ }^{2}$ ) auf unsere Veranlassung noch die $O$-Benzoylirung des Dibenzoylmethans ausgefübrt. Sie kann leicht durch die Behandlung dieses Diketons mit Benzoylchlorid und Pyridin bewirkt werden, während die Einwirkung von Benzoylchlorid auf das Natriumsalz des. Dibenzoylmethans nach den früberen Untersuchungen von Claisen ${ }^{3}$ ), ausschliesslich das $C$-Benzoylderivat, d. b. Tribenzoylmethan, ergiebt: CO. $\mathrm{C}_{6} \mathrm{H}_{5}$

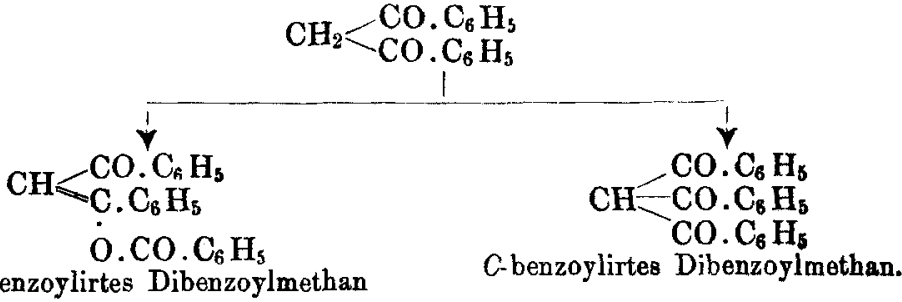

1) In ähnlicher Weise könnte, mit einer geringen Modification der früberen. Ansicht. auch die Natracetessigestersy these als in den folgenden Pbasen verlaufend gedacht werden: 1. Entstehung von $\mathrm{CH}_{3} . \mathrm{C}\left(\mathrm{OC}_{2} \mathrm{H}_{5}\right)_{2} \mathrm{ONa}$; 2. Uebergang desselben durch Alkoholabgabe in $\mathrm{CH}_{2}: \mathrm{C}\left(\mathrm{OC}_{2} \mathrm{H}_{5}\right) \mathrm{ONa}$; 3. Addition des. Letzteren in derselben Weise, wie es früher für das Natriumäthylat angenommen wurde, an das Carbonyl eines zweiten Moleküls Essigester; 4. Umlagerung des so gebildeten Complexes unter Alkoholaustritt (ähnlich wie in den obigen Schemata) in Natracetessigester. - Die zur Zeit von Nef geausserte Ansicht, dass an intermediär entstandenes $\mathrm{CH}_{2}: \mathrm{C}\left(\mathrm{OC}_{2} \mathrm{H}_{5}\right) \mathrm{ONa}$ Essigester sich in Form von $\mathrm{H}$ und $. \mathrm{CH}_{2} . \mathrm{COOC}_{2} \mathrm{H}_{5}$ addirt, ist völlig unbaltbar, weil diese Erklärung bei anderen, zweifellos analogen Vorgängen (z. B. bei der Condensation von Benzoëester mit Essigester zu Benzoylessigester) versagt.

2) Vergl. dessen Inauguraldissertation, Kiel 1901, S. 34.

3) Ann. d. Chem. 291, 90. 
O-Benzoat des Dibenzoylmethans. Dibenzoylmethan (1 Mol.) wurde in Pyridin (2 Mol.) gelöst und nach Zusatz ron Benzoylchlorid (2 Mol.) 2 Stunden auf dem Wasserbade erwärmt. Anf nachherige Zugabe von Eiswasser und Aether schied sich ein gelber, krystallinischer Körper aus, welcher abgesaugt und mit verdünnter Natronlauge gewaschen wurde. Durch Umkrystallisiren aus wenig heissem Alkohol wurde er in schwefelgelben Blättchen und kleinen Täfelchen vom Schmp. $108-109^{\circ}$ erhalten. Er ist unlöslich in verdünnten Alkalien und wird in alkoholischer Lösung von Eisenchlorid nicht gefürbt. Die Ausbente betrug $80-90 \mathrm{pCt}$. der Theorie.

$0.2228 \mathrm{~g}$ Sbst.: $0.6559 \mathrm{~g} \mathrm{CO}_{2}, 0.0989 \mathrm{~g} \mathrm{H}_{2} \mathrm{O} .-0.129 \mathrm{~g}$ Sbst: $0.3793 \mathrm{~g}$ $\mathrm{CO}_{2}, 0.0562 \mathrm{~g} \mathrm{H}_{2} \mathrm{O}$.

$$
\begin{array}{ll}
\mathrm{C}_{22} \mathrm{H}_{16} \mathrm{O}_{3} . & \begin{array}{l}
\text { Ber. C } 80.49, \\
\text { Gef. } \$ 80.28,80.20, 》 4.93,
\end{array} \\
& \text { H } 4.84 .
\end{array}
$$

Wird dieses Benzoat mehrere Standen auf $230-240^{\circ}$ erhitzt, so sublimirt Benzoësäure beraus, und es hinterbleibt eine Substanz, die sich durch Digeriren mit warmem Alkohol und Umkrystallisiren des Ungelösten aus sıedendem Aceton in sehr kleinen, weissen Kryställchen vom ungefähren Schmp. $225-230^{\circ}$ gewinnen lässt. Wir batten diesen Körper früher für Tribenzoylmethan gehalten, welches bei annähernd derselben Temperatur schmilzt ${ }^{1}$ ) und sich ja auch in diesem Falle durch Verschiebung des Benzoyls vom Sauerstoff an den Koblenstoff leicht hätte bilden können. Da eine diesbezügliche Notiz auf Grund einer brieflichen Mittheilung in eine Abhandlung von W. $W_{\text {islicenus }}^{2}$ ) übergegangen ist, möchten wir nicht säumen, die Sache dahin richtig zu stellen, dass der fragliche Körper die Zusammensetzung des Tribenzoylmethans ( $80.49 \mathrm{pCt}$. C und $4.88 \mathrm{pCt}$. H) vicht besitzt; bei mehreren Analysen wurden im Mittel $86.90 \mathrm{pCt}$. C nnd 5.10 pCt. H gefunden. Die Substanz scbeint hiernach aus dem $\mathrm{Di}$ benzoylmethan- $O$-Benzoat durch Austritt von einem Molekül Benzoësäure entstanden za sein; die durch diesen Vorgang sich ergebende Formel $\mathrm{C}_{15} \mathrm{H}_{10} \mathrm{O}^{3}$ ) würde $87.38 \mathrm{pCt}$. $\mathrm{C}$ und $4.85 \mathrm{pCt}$. H verlangen.

Dureh die im Vorstehenden mitgetheilten Versuche ist die Reihe der Körper, welche ans dem monobenzoylirten Methan - dem Acetophenon - durch fortschreitende Benzoylirung entstehen, zum $\mathbf{A b}$ schluss gekommen. Wie man sieht, hat dieses anscheinend so einfache Problem sich immer mehr complicirt, je tiefer man in die diesbezüglichen Verbültnisse eingedrungen ist. Noch vor etwa 12 Jahren,

1) Ann. d. Chem. 291, 93. 2) Diese Berichte 34, 218 [1901].

3) Oder eines Polymeren $\left(\mathrm{C}_{15} \mathrm{H}_{10} \mathrm{O}\right.$. 
ehe der Eine von uns an die Untersuchungen über die Acylirung der Ketone herantrat. hielt man mit der Reihe:

I. $\mathrm{CH}_{3} \cdot \mathrm{CO} \cdot \mathrm{C}_{6} \mathrm{H}_{5}$, Monobenzoylmethan;

II. $\mathrm{CH}_{2}\left(\mathrm{CO} . \mathrm{C}_{6} \mathrm{H}_{5}\right)_{2}$, Dibenzoylmethan;

III. $\mathrm{CH}\left(\mathrm{CO} . \mathrm{C}_{6} \mathrm{H}_{5}\right)_{3}$, Tribenzoylmethan;

IV. $\mathrm{C}\left(\mathrm{CO} . \mathrm{C}_{6} \mathrm{H}_{3}\right)_{4}$, Tetrabenzoylmethan,

die Zahl der Benzoylderirate des Methans für erschöpft. Jetzt weiss man - oder muss es doch nach den zahlreichen, auf seine Darstellung gerichteten Versuchen für sehr wahrscheinlich halten -, dass Körper IV (das eigentliche Tetrabenzoylmethan) überhaupt nicht existenzfähig ist ${ }^{1}$ ). Andererseits hat sich die Zahl der ans dem Acetophenon durch fortschreitende Benzoylirung gewinnbaren Derivate ron den damals angenommenen dreien auf sechs erhöht. Durch $\mathrm{zwei}$ von einander anabhängige Ursachen wird diese Complication hervorgernfen: 1. dadurch, dass das Benzoyl, das in das mono-, di- oder tri-benzoylirte Methan neu eintritt, sich eutweder an den Sauerstoff oder an den Kohlenstoff begeben kann (Gegensatz von $O$-Benzoyl- und $C$-Benzoyl-Derivat); 2. durch die von Clais $\mathrm{n}^{2}$ ) entdeckte Differenzirung der Di- und TriKetone in die $\alpha$ - und $\beta$-Formen (Gegensatz von Diketo- bezw. TriketoForm zu Ketoenol- bezw. Diketoenol-Form). Unter Beachtung dieser beiden Gesichtspunkte lassen sich die im vorliegenden Falle gegebenen Isomerien leicht und übersichtlich in folgender Weise entwickeln:

I. Monobenzoylmethan (Acetophenon).

In freiem Zustand nur als Keton, $\mathrm{CH}_{3} . \mathrm{CO} . \mathrm{C}_{6} \mathrm{H}_{5}$, nicht als Enol, bekannt. Die Enolform $\mathrm{CH}_{2}: \mathrm{C}(\mathrm{OH}), \mathrm{C}_{6} \mathrm{H}_{5}$ ist nur in Derivaten vertreten, nämlich 1 . in den Claisen'schen Alkyläthern ${ }^{3}$ ), z. B. $\mathrm{CH}_{2}: \mathrm{C}\left(\mathrm{OC}_{2} \mathrm{H}_{3}\right) \cdot \mathrm{C}_{6} \mathrm{H}_{5}$, und 2. in Acylderivaten, z. B. dem Leesschen O-Benzoat, $\mathrm{CH}_{2}: \mathrm{C}\left(\mathrm{O} \cdot \mathrm{CO} \cdot \mathrm{C}_{6} \mathrm{H}_{5}\right) \cdot \mathrm{C}_{6} \mathrm{H}_{5}$.

II. Benzoylirtes Monobenzoylmethan.

Die Benzoylirung führt, je nach den Bedingungen, zu dem $O$ Benzoyl- oder $C$-Benzoyl-Derivat:

a) $\mathrm{CH}_{2}: \mathrm{C} \cdot \mathrm{C}_{6} \mathrm{H}_{5}$

$O$-benzoylirtes Acetophenon. b) $\mathrm{CH}_{2}<\mathrm{CO} \mathrm{CO}_{6} \mathrm{C}_{6} \mathrm{H}_{5}$ $C$-benzoylirtes Acetophenon (Dibenzoylmethan).

1) Was wohl überhaupt für die tetraacylirten Methane, $C(C O . R)_{1}$, gilt. Auch für das meist als $\mathrm{C}\left(\mathrm{NO}_{9}\right)_{4}$ betrachtete $\mathrm{T}$ etranitromethan scheint nach dessen Verhalten die Formel

nicht ganz ausgeschlossen.

$$
\begin{aligned}
& \mathrm{C} \leftarrow \mathrm{NO}_{2} \\
& \mathrm{NO} \mathrm{NO}_{2} \\
& \mathrm{NO} . \mathrm{NO}_{2}
\end{aligned}
$$

2) Diese Berichte 27, 114 [1894]; Ann. d. Chem. 277, 184, und 291, 25.

3) Diese Berichte 29, 1006 und 2932 [1896]; 31, 1020 [1898]. 
a) Aus Acetophenon durch Kochen mit Benzoylchlorid; b) ans A cetophenon durch Behandlung mit Natrium und Benzoëester. a) kann durch Verschiebung des Benzoyls in b) umgelagert werden.

\section{Benzoylirtes Dibenzoylmethan.}

Die $C$-Benzoylirung (Einwirkung von Benzoylehlorid auf NatriumDibenzoylmethan) ergiebt Tribenzoylmethan, von welchem $\mathrm{Claisen}{ }^{1)}$ neben der schon länger bekannten $\beta$-Form (Triketoform) noch die sehr labile $\alpha$-Form (Diketoenolform) kennen lehrte:

a)

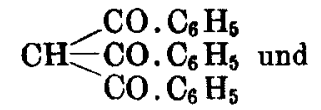
$\mathrm{CO} . \mathrm{C}_{6} \mathrm{H}_{3}$ $\mathrm{CO} . \mathrm{C}_{6} \mathrm{H}_{5}$ $\mathrm{C}(\mathrm{OH}) \cdot \mathrm{C}_{6} \mathrm{H}_{5}$

Das diesen beiden isomere Dibenzoylmethan-O-Benzoat,

$$
\text { c) } \begin{aligned}
& \mathrm{CH} \leqslant \mathrm{CO} \cdot \mathrm{C}_{\mathrm{R}} \mathrm{H}_{5} \\
& \text { O. } \mathrm{C}_{6} \mathrm{H}_{5} \\
& \text { O. CO. } \mathrm{C}_{6} \mathrm{H}_{5}
\end{aligned}
$$

wird glatt bei der Behandlung des Diketons mit Pyridin und Benzoylchlorid gebildet. Die Umlagerung des Letzteren in Tribenzoylmethan ist noch nicht ausgeführt worden, dürfte aber nach bekannten Analogien (vergl. die Umlagerung der $O$-acylirten Ketonsäureester in die $C$-acylirten) durch passende Reactionen wohl zu bewirken sein.

\section{Benzoylirtes Tribenzoylmethan.}

Aus Tribenzoylmethan wird bei der Benzoylirung unter allen Bedingungen (also auch bei Anwendung des Natriumsalzes) das o.Benzoat,

$$
\begin{gathered}
\mathrm{CO}-\mathrm{C}_{6} \mathrm{H}_{5} \\
\mathrm{CO} . \mathrm{C}_{6} \mathrm{H}_{5} \\
\mathrm{C} . \mathrm{C}_{6} \mathrm{H}_{5} \\
\text { O. } \mathrm{CO} . \mathrm{C}_{6} \mathrm{H}_{5}
\end{gathered}
$$

nie das isomere $C$-Benzoylderivat [das eigentliche Tetrabenzoylmethan, $\left.\mathrm{C}\left(\mathrm{CO}, \mathrm{C}_{6} \mathrm{H}_{6}\right)_{4}\right]$ gebildet. Das Letztere scheint daher, wie schon bemerkt, nicht existenzfähig zu sein.

Im ganzen bestätigen diese Verbältnisse die frühere Regel $\left.{ }^{\natural}\right)$, dass bei den acylirten Methanen, $\mathrm{CH}_{3}(\mathrm{CO} . \mathrm{K}), \mathrm{CH}_{2}(\mathrm{CO} . \mathrm{R})_{2}$ und $\mathrm{CH}(\mathrm{CO} . \mathrm{R})_{3}$, die Neigung zur Bildung der Enolform bezw. von Derivaten derselben mit der Zabl der vorbandenen Acyle wächst.

Ungewiss bleibt nur noch die Formel des Dibenzoylmethans, von dem sich (wie bei den meisten 1.3-Diketonen) nicht mit Sicher- 
heit sagen lässt, ob es $\mathrm{C}_{6} \mathrm{H}_{5} \cdot \mathrm{CO} \cdot \mathrm{CH}_{2} \cdot \mathrm{CO} \cdot \mathrm{C}_{6} \mathrm{H}_{5}$ oder $\mathrm{C}_{6} \mathrm{H}_{5} . \mathrm{C}(\mathrm{OH})$ :CH.CO. $\mathrm{C}_{6} \mathrm{H}_{5}$ ist. Von erheblichem Interesse für diese Frage sind die vor einigen Jabren erfolgten Mittheilnngen von J. Wislicenus ${ }^{1}$ ) über ein neues "Dibenzoylmethan", welches aus Benzylidenacetopbenondibromid bei der Behandlung mit alkobolischem Kali entsteht. Es hat denselben Schmelz- und Siede-Punkt, wie das schon länger bekannte Isomere und kann durch zahlreiche Reactionen in dieses oder in Derivate desselben umgewandelt werden, während der umgekehrte Uebergang anscheinend nicht möglich ist. Hauptunterschied ist. das Verhalten gegen Eisenchlorid - die neue Substanz giebt damit keine Färbung - und gegen verdünnte, wässrige Alkalien, von denen nur das »alte« Dibenzoylmethan, nicht das "neve«, gelöst wird. Könnte man hiernach geneigt sein, zwischen den beiden Körpern die Formelbeziehung

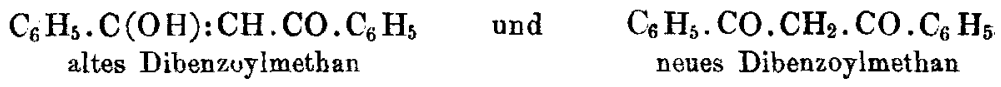

anzunehmen, so zeigen sich wieder andere $Z$ üge, die nicht recht in das Bild, das man bis jetzt von dem Verhalten solcher Isomerenpaare gewonnen hat, hineinpassen. Auffallend z. B. ist, dass jeder der beiden Körper völlig unverändert destillirt, während in den sonstigen Fällen durch Erwärinen eine Umwandlang nach der einen oder der anderen Richtung hervorgerufen wird. Deshalb glaubt J. Wislicenus, für das neve Isomere neben der obigen Formel auch die eines Phenylbenzoyläthylenoxyds, $\mathrm{C}_{6} \mathrm{H}_{5}$. CH.CH.CO. $\mathrm{C}_{6} \mathrm{H}_{5}$, in Betracht ziehen zo ó

sollen. Klarheit hierüber wird erst durch weitere Untersuchungen $z \mathbf{x}$ gewinnen sein.

\section{O. Hauser und L. Vanino: Ueber das Wismuth- chloridpyridin.}

(Eingegangen am 28. October 1903.)

Vor längerer Zeit beschrieben wir einige Versuche über Verbindungen des Wismuthchlorids mit Basen der aromatischen Reibe. Diese Verbindungen sind im allgemeinen schwer löslich, zeigen starke, hydrolytische Empfindlicbkeit, und ihre complexe Natur ist nur wenig

1) J. Wislicenus, Ann. d. Chem. 308, 219 und die Inauguraldissertationen von Löwenheimer und Wells, Leipzig 1890 und 1897. 